\title{
Fractal Theory and Contact Dynamics Modeling Vibration Characteristics of Damping Blade
}

\author{
Ruishan Yuan, Qin Zhou, Qiang Zhang, and Yonghui Xie \\ School of Energy and Power Engineering, Xi'an Jiaotong University, Xian 710049, China \\ Correspondence should be addressed to Yonghui Xie; yhxie@mail.xjtu.edu.cn
}

Received 27 February 2014; Accepted 16 March 2014; Published 7 April 2014

Academic Editor: Xiao-Jun Yang

Copyright ( 2014 Ruishan Yuan et al. This is an open access article distributed under the Creative Commons Attribution License, which permits unrestricted use, distribution, and reproduction in any medium, provided the original work is properly cited.

\begin{abstract}
The contact surface structure of dry friction damper is complicate, irregular, and self-similar. In this paper, contact surface structure is described with the fractal theory and damping blade is simplified as 2-DOF cantilever beam model with lumped masses. By changing the position of the damper, lacing and shroud structure are separately simulated to study vibration absorption effect of damping blade. The results show that both shroud structure and lacing could not only dissipate energy but also change stiffness of blade. Under the same condition of normal pressure and contact surface, the damping effect of lacing is stronger than that of shroud structure. Meanwhile, the effect on changing blade stiffness of shroud structure is stronger than that of lacing. This paper proposed that there is at least one position of the blade, at which the damper dissipates the most vibration energy during a vibration cycle.
\end{abstract}

\section{Introduction}

Lacing and shroud structure are widely adopted in turbine blades to effectively restrain blade resonance and have features such as simple structure and easy installation. Lacing and shroud structure are based on the mechanism of dry friction between adjacent blades to achieve lower blade vibration. The blades are pretwistedly installed so that the lacing and shroud structure among adjacent blades come into contact and generate some positive pressure. In operation, blades twisted under the centrifugal force and the positive pressure caused by torsion provide adequate positive pressure for adjacent blades.

While forced by the exciting force, blades vibrate and the relative displacements among adjacent blades are formed. The dry friction structures of lacing and shroud, on the one hand, dissipate vibration energy. On the other hand, the structure not only changes the mass of the blade but also changes the stiffness of the blade so that the inherent frequency and the exciting force frequency of the blade could avoid each other well. Thus the purpose of reducing the vibration level of the blade is achieved.

The contact surface structure of dry friction damper can be described with the fractal theory accurately. Since the fractal theory was proposed by Benoit B. Mandelbrot in
1975, it has been widely applied. Based on fractal dimension, Ritchie and Olff [1] proposed biodiversity calculation model by researching the relationship between space scaling relation and biodiversity. Taylor et al. [2] studied Pollock's watercolors by using the fractal theory.

Using the dry friction model to inhibit vibration is a very effective method. The famous scholars Den Hartog [3] proposed the Coulomb model. After fully developing the Coulomb model and intensively studying dry friction, Meng et al. did further research on two-dimensional sliding movement on the basis of the microscopic sliding study in $1991[4,5]$. Muszynska et al. [6-8] simplified the blade and damper structure using the centralized quality model of multidegrees of freedom. Csaba [9] then combined the onebar microslip model with the centralized quality model of two degrees of freedom to study the blade with a damper. Lui et al. [10] and others proposed a new model to research the dry friction under different roughness and positive pressure condition.

In order to accurately describe contact interface and reveal the vibration reduction mechanisms of lacing and shroud structure, a centralized quality cantilever model of two degrees of freedom is built using the fractal theory in this paper. By placing the damper on different masses, the vibration reduction effect of lacing and shroud structure 
is simulated. By using the one-bar microslip model, the constitutive equation of the force among friction surface is established. The time-frequency domain interactive method is adopted in the solving process of lacing and shroud structure. Therefore, the corresponding results at the steady state are obtained.

\section{The One-Bar Microslip Model}

For damping blade, the damper structure and boundary conditions are very complex. The contact interface, especially, consists of a series of concave and convex structure in different sizes. It has high self-similarity and it is difficult to describe the structure using the same size. In order to develop a model as simple as possible but also complete enough to show the most important properties of friction interface, the contact interface is described using fractal dimension and fractal length in this paper. Fractal dimension can be calculated by a variety of methods. And similarity dimension method is adopted here.

Assuming objects or geometric shapes can be divided into $N$ parts, each part is similar to the whole object with a likelihood ratio $\beta$. The fractal dimension can be expressed as

$$
D=\frac{\ln N}{\ln (1 / \beta)}=-\frac{\ln N}{\ln \beta} .
$$

The fractal dimension $D$ does not have to be an integer.

The fractal curve length estimation model given by Mandelbrot is expressed as

$$
L=L_{0} \varepsilon^{1-D},
$$

where $L$ is the fractal curve's Euclidean length; $L_{0}$ is the initial operation length of fractal curve; $\varepsilon$ is the scale of fractal curve; $D$ is the fractal dimension.

The damper is modelled by a rectangular bar pressed against a rigid surface with a normal load $q$ and subjected to a force $F$, shown in Figure 1. The normal load on the bar is assumed to be constant over the width of the bar and defined by a quadratic normal load function in the lengthwise direction:

$$
q(x)=q_{0}+q_{2} \frac{4\left(x l-x^{2}\right)}{l^{2}} .
$$

The force $F$ can be expressed as

$$
F=F_{a} \sin \omega t
$$

The bar has a modulus of elasticity $E$ and a cross section area $A$. The length of the bar is $l$, and the coefficient of friction $\mu$ is assumed to be constant across the contact zone and independent whether the bar is sliding or not.

The bar starts to slip, as the force is applied. Then the bar may be divided into two zones, one that is slipping and one that is stuck. The length of the slip zone is defined as the slip length $\delta$ and the slip length corresponding to the maximum force $F_{\text {amp }}$ is $\delta_{a}$, shown in Figure 2(a).

The zone that is slipping is stretched after the initial loading with $F=F_{\mathrm{amp}}$. As the force decreases from $F_{\mathrm{amp}}$ to

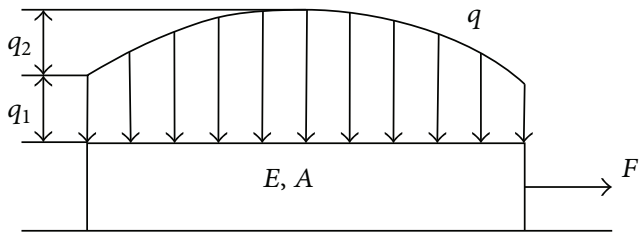

FIgURE 1: Microslip model for the friction interface.

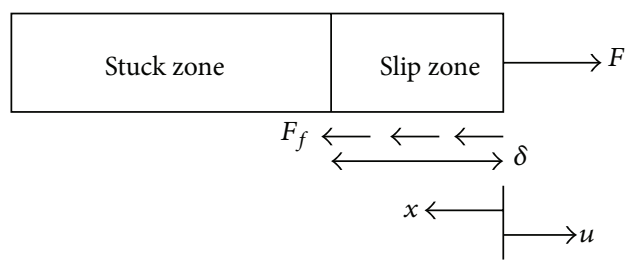

(a) Stuck and slip zones when initially loading the bar

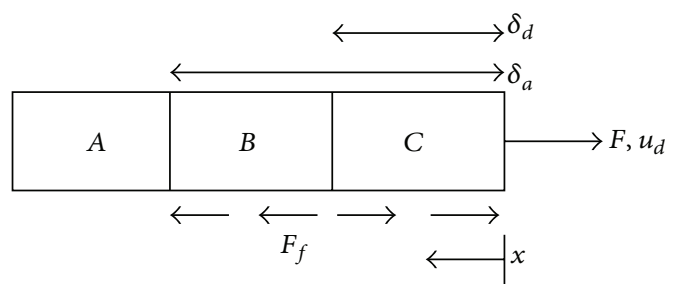

(b) Illustrative plot of force and displacement when $F$ is decreasing

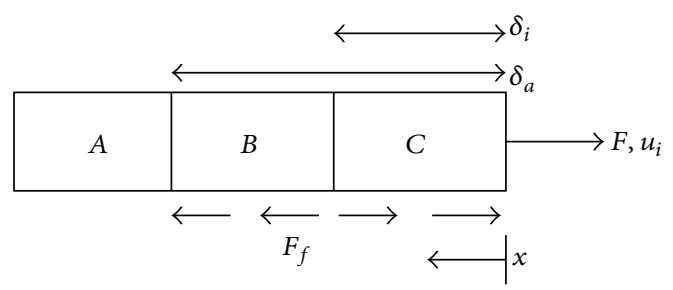

(c) Illustrative plot of force and displacement when $F$ is increasing

FIGURE 2: Microslip model for damper.

$-F_{\text {amp }}$, the bar may be divided into three zones in this analysis, which is shown in Figure 2(b).

Zone $A$ is stuck and has zero strain.

Zone $B$ is stuck and stretched.

Zone $C$ is slipping and compressed.

The length of the compression zone is denoted as the slip length $\delta_{d}$. Zone $C$ will increase and zone $B$ will decrease as $F$ decreases. This will continue until $F=-F_{\text {amp }}$. Zone $B$ is then eliminated and $\delta_{d}$ equals $\delta_{a}$.

Here we have the opposite situation as in the previous part. As the force increases from $-F_{\text {amp }}$ to $F_{\text {amp }}$, the bar may be divided into three zones in this analysis, which is shown in Figure 2(c).

Zone $A$ is stuck and has zero strain.

Zone $B$ is stuck and compressed.

Zone $C$ is slipping and stretched. 
The length of stretched zone is denoted as the slip length $\delta_{i}$. Zone $C$ will increase and zone $B$ will decrease as $F$ increases. This will continue until $F=F_{\text {amp }}$. Zone $B$ is then eliminated and $\delta_{d}$ equals $\delta_{a}$.

The length of the slip zone is defined as the slip length $\delta$. The slip length corresponding to the maximum force $F_{\text {amp }}$ is $\delta_{a}$. The displacement of the bar is defined as $u$. According to the mechanics analysis, it can be seen that force $F$ and displacement $u$ are the function of slip length $\delta$.

As the force is applied, the force at the right bar end $F$ and displacement can be expressed as

$$
\begin{aligned}
& F(\delta)=\int_{0}^{\delta} F_{f}(x) d x=\mu q_{0} \delta+\frac{2 \mu q_{2}}{3 l^{2}}\left(3 \delta^{2} l-2 \delta^{3}\right), \\
& u(\delta)=\frac{\mu q_{0} \delta^{2}}{2 E A}+\frac{2 \mu q_{2}}{3 E A l} 2 \delta^{3}-\frac{\mu q_{2}}{3 E A l^{2}} 3 \delta^{4} .
\end{aligned}
$$

As the force decreases from $F_{\mathrm{amp}}$ to $-F_{\mathrm{amp}}$, the force at the right bar end $F$ and displacement can be expressed as

$$
\begin{aligned}
F_{d}\left(\delta_{a}, \delta_{d}\right)= & \mu q_{0}\left(\delta_{a}-2 \delta_{d}\right) \\
& +\frac{2 \mu q_{2}}{3 l^{2}}\left(3 l \delta_{a}^{2}-2 \delta_{a}^{3}+4 \delta_{d}^{3}-6 l \delta_{d}^{2}\right), \\
u_{d}\left(\delta_{a}, \delta_{d}\right) & \\
= & \frac{\mu}{E A}\left(q_{0} \frac{\delta_{a}^{2}-2 \delta_{d}^{2}}{2}+q_{2}\left(\frac{4\left(\delta_{a}^{3}-2 \delta_{d}^{3}\right)}{3 l}-\frac{\delta_{a}^{4}-2 \delta_{d}^{4}}{l^{4}}\right)\right) .
\end{aligned}
$$

As the force decreases from $-F_{\text {amp }}$ to $F_{\text {amp }}$, the force at the right bar end $F$ and displacement can be expressed as

$$
\begin{aligned}
& F_{i}\left(\delta_{a}, \delta_{i}\right)=\mu q_{0}\left(2 \delta_{i}-\delta_{a}\right) \\
& +\frac{2 \mu q_{2}}{3 l^{2}}\left(2 \delta_{a}^{3}-3 l \delta_{a}^{2}+6 l \delta_{i}^{2}-4 \delta_{i}^{3}\right), \\
& u_{i}\left(\delta_{a}, \delta_{d}\right) \\
& =\frac{\mu}{E A}\left(q_{0} \frac{2 \delta_{i}^{2}-\delta_{d}^{2}}{2}+q_{2}\left(\frac{4\left(2 \delta_{i}^{3}-\delta_{a}^{3}\right)}{3 l}-\frac{2 \delta_{i}^{4}-\delta_{a}^{4}}{l^{2}}\right)\right) .
\end{aligned}
$$

Tow linearization techniques are discussed to transform the nonlinear properties of friction into equivalent damping and stiffness. Two criteria are established in Lazan's linearization method, for equivalence of the two loops.

(a) The same value of loop area is the same as damping energy per cycle $W$.

(b) The amplitude of force $F_{\text {amp }}$ and displacement $u_{\text {amp }}$ should be the same.

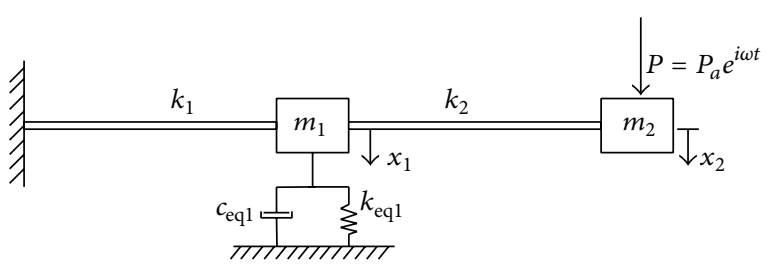

Figure 3: Simplified damping blade with lacing structure.

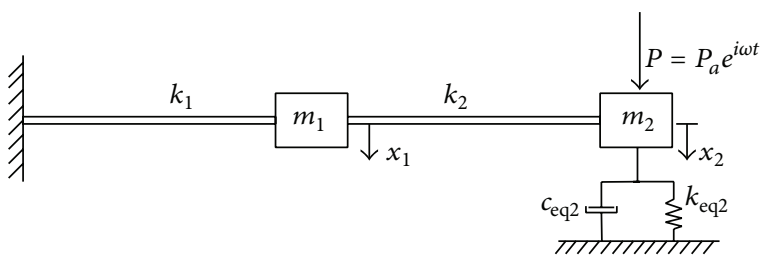

FIgURe 4: Simplified damping blade with shroud structure.

The first criterion gives the equivalent viscous damping $c_{\text {eq }}$, while the second criterion together with $c_{\text {eq }}$ gives the equivalent stiffness $k_{\text {eq }}$ :

$$
\begin{aligned}
& w\left(\delta_{a}\right)=\frac{2 \mu^{2} \delta_{a}^{3}}{3 E A}\left[q_{0}^{2}+\left(\frac{4 \delta_{a}}{l}-\frac{14 \delta_{a}^{2}}{5 l^{2}}\right) q_{0} q_{2}\right. \\
& \left.+\left(\frac{8 \delta_{a}^{4}}{7 l^{4}}-\frac{4 \delta_{a}^{3}}{l^{3}}+\frac{16 \delta_{a}^{2}}{5 l^{2}}\right) q_{2}^{2}\right], \\
& c_{\mathrm{eq}}=\frac{2 \mu^{2} \delta_{a}^{3}}{3 E A \pi \omega u_{\mathrm{amp}}^{2}}\left[q_{0}^{2}+\left(\frac{4 \delta_{a}}{l}-\frac{14 \delta_{a}^{2}}{5 l^{2}}\right) q_{0} q_{2}\right. \\
& \left.+\left(\frac{8 \delta_{a}^{4}}{7 l^{4}}-\frac{4 \delta_{a}^{3}}{l^{3}}+\frac{16 \delta_{a}^{2}}{5 l^{2}}\right) q_{2}^{2}\right], \\
& k_{\text {eq }}=\sqrt{\left(\frac{F_{\text {amp }}}{u_{\text {amp }}}\right)^{2}-\left(\omega c_{\text {eq }}\right)^{2}} .
\end{aligned}
$$

\section{The Forced Vibration Response Analysis of Damped Blade}

A two-degree freedom system simplifying the blade is used for forced vibration response analysis in this paper. The 2DOF system is built up of a massless beam, two concentrated masses, and the damper. Compared with the previous study, this paper simulates lacing and shroud structure by locating damper on $m_{1}$ and $m_{2}$, respectively, and the structure is shown in Figures 3 and 4 . The damper is described by a viscous damper and spring, $c_{\text {eq } 1}$ and $k_{\text {eq } 1}$ and $c_{\text {eq } 2}$ and $k_{\text {eq } 2}$, respectively. Displacements where the damper is attached and the force is applied are $x_{1}$ and $x_{2}$, respectively.

The following parameters of the whole system are as follows, assuming that $Q_{0}=\mu q_{0} l / P_{a}$ :

$$
\begin{aligned}
& \text { beam system } k_{1}=1.0 \times 10^{7} \mathrm{~N} / \mathrm{m}, k_{2}=1.0 \times 10^{7} \mathrm{~N} / \mathrm{m}, \\
& m_{1}=0.05 \mathrm{~kg}, \text { and } m_{2}=0.05 \mathrm{~kg} ;
\end{aligned}
$$


damper system $E_{1} A_{1}=40000 \mathrm{~N}, l=0.2 \mathrm{~m}$, and $Q_{2}=$ $Q_{0} / 2$.

The equations of motion for the system are

$$
\begin{aligned}
m_{1} x_{1} \ddot{(}(t) & +c_{\mathrm{eq} 1} x_{1} \dot{(}(t) \\
& +\left(k_{1}+k_{2}+k_{\mathrm{eq} 1}\right) x_{1}(t)-k_{2} x_{2}(t)=0, \\
& m_{2} \ddot{x}_{2}(t)+k_{2} x_{2}(t)-k_{2} x_{1}(t)=P_{a} e^{i \omega t} .
\end{aligned}
$$

Assuming that harmonic motion yields

$$
x_{1}(t)=x_{1 a} e^{i \omega t} x_{2}(t)=x_{2 a} e^{i \omega t},
$$

we define the complex stiffness as

$$
K_{1 a}=k_{\mathrm{eq} 1}+i \omega c_{\mathrm{eq} 1} .
$$

Solving equations for $x_{1}$ and $x_{2}$ yields

$$
\begin{aligned}
x_{1}= & \frac{P_{a} k_{2}}{\left(k_{2}-m_{2} \omega^{2}\right)\left(k_{1}+k_{2}+K_{1 a}-m_{1} \omega^{2}\right)-k_{2}^{2}}, \\
x_{2}= & \frac{P_{a}}{\left(k_{2}-m_{2} \omega^{2}\right)} \\
+ & \left(\left(P_{a} k_{2}^{2}\right)\right. \\
& \quad \times\left(\left(\left(k_{2}-m_{2} \omega^{2}\right)\left(k_{1}+k_{2}+K_{1 a}-m_{1} \omega^{2}\right)-k_{2}^{2}\right)\right. \\
& \left.\left.\quad \times\left(k_{2}-m_{2} \omega^{2}\right)\right)^{-1}\right) .
\end{aligned}
$$

The equations of motion for the system are

$$
\begin{gathered}
m_{1} \ddot{x}_{1}(t)+\left(k_{1}+k_{2}\right) x_{1}(t)-k_{2} x_{2}(t)=0, \\
m_{2} x_{2} \ddot{(t)}+c_{\mathrm{eq} 2} x_{2}(t)+\left(k_{2}+k_{\mathrm{eq} 2}\right) x_{2}(t)-k_{2} x_{1}(t) \\
=P_{a} e^{i \omega t} .
\end{gathered}
$$

Assuming that harmonic motion yields

$$
x_{1}(t)=x_{1 a} e^{i \omega t} x_{2}(t)=x_{2 a} e^{i \omega t},
$$

we define the complex stiffness as

$$
K_{1 a}=k_{\mathrm{eq} 2}+i \omega c_{\mathrm{eq} 2} .
$$

Solving equations for $x_{1}$ and $x_{2}$ yields

$$
\begin{aligned}
x_{1}= & \frac{P_{a} k_{2}}{\left(k_{2}+K_{2 a}-m_{2} \omega^{2}\right)\left(k_{1}+k_{2}-m_{1} \omega^{2}\right)-k_{2}^{2}}, \\
x_{2}= & \frac{P_{a}}{\left(k_{2}+K_{2 a}-m_{2} \omega^{2}\right)} \\
& +\left(\left(P_{a} k_{2}^{2}\right)\right. \\
& \quad \times\left(\left(\left(k_{2}+K_{2 a}-m_{2} \omega^{2}\right)\left(k_{1}+k_{2}-m_{1} \omega^{2}\right)-k_{2}^{2}\right)\right. \\
& \left.\left.\quad \times\left(k_{2}+K_{2 a}-m_{2} \omega^{2}\right)\right)^{-1}\right) .
\end{aligned}
$$

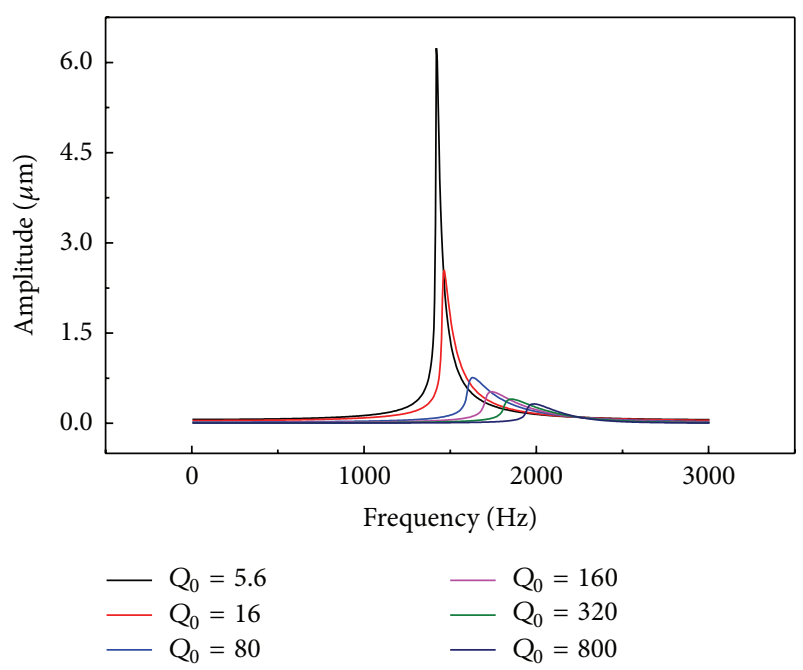

FIGURE 5: Vibration response of dry friction blade $m_{1}$ with lacing.

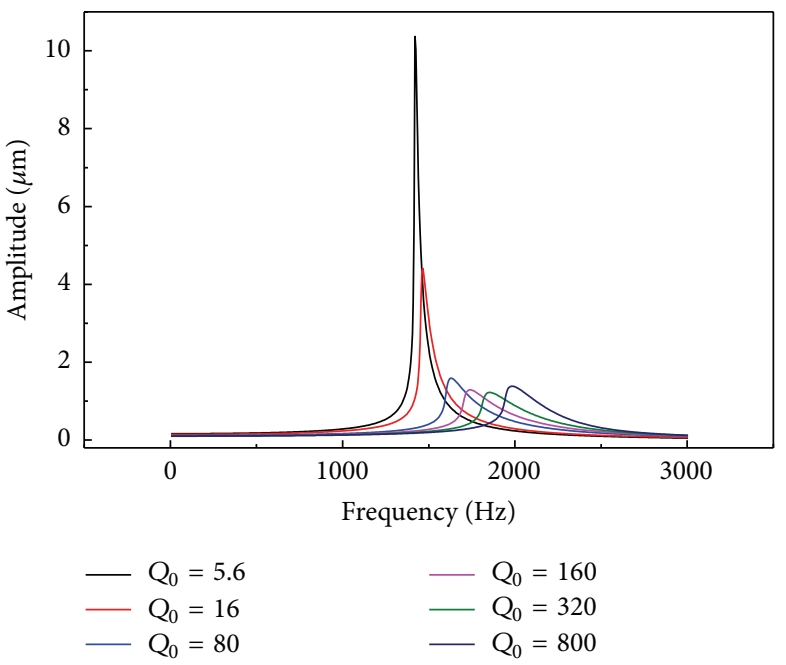

FIgURE 6: Vibration response of dry friction blade $m_{2}$ with lacing.

\section{Results and Discussion}

Through blades vibration analysis with lacing in Figure 3, amplitude-frequency response curves of $m_{1}$ and $m_{2}$ in the range of $0 \sim 3000 \mathrm{~Hz}$ are achieved. The results are shown in Figures 5 and 6. Different positive pressure and damper parameters under different resonance states are shown in Table 1.

From Figures 5 and 6, it is obvious that, when the damper is placed on the mass $m_{1}$ to simulate lacing structure, the resonance amplitude of $m_{1}$ decreases with the increase of the positive pressure. On the contrary, the resonance frequency of $m_{1}$ increases with the increase of positive pressure. It could also be seen that, with the increase of positive pressure, the equivalent stiffness increases. From the vibration mechanics, the resonant frequency is positively correlated with the system stiffness as the resonance amplitudes negatively 
TABLE 1: Resonance parameters of lacing damper.

\begin{tabular}{lccccc}
\hline Positive pressure $Q_{0}$ & $u_{\text {amp }}(\mu \mathrm{m})$ & $F_{\text {amp }}(\mathrm{N})$ & $\begin{array}{c}\text { Resonance } \\
\text { frequency }(\mathrm{Hz})\end{array}$ & $\begin{array}{c}\text { Equivalent viscous } \\
\text { damping }(\mathrm{N} \cdot \mathrm{s} / \mathrm{m})\end{array}$ & $\begin{array}{c}\text { Equivalent stiffness } \\
(\mathrm{N} / \mathrm{s})\end{array}$ \\
\hline 5.6 & 6.23854 & 4.217 & 1420 & 176 & 676039 \\
16 & 2.55285 & 4.309 & 1465 & 453 & 1687830 \\
80 & 0.75866 & 5.022 & 1630 & 1689 & 6619180 \\
160 & 0.52390 & 5.858 & 1740 & 2696 & 11180741 \\
320 & 0.40169 & 7.223 & 1855 & 4085 & 17980921 \\
800 & 0.32137 & 10.183 & 1985 & 6750 & 31686540 \\
\hline
\end{tabular}

TABLE 2: Resonance parameters of shroud structure damper.

\begin{tabular}{lccccc}
\hline Positive pressure $Q_{0}$ & $u_{\text {amp }}(\mu \mathrm{m})$ & $F_{\text {amp }}(\mathrm{N})$ & $\begin{array}{c}\text { Resonance } \\
\text { frequency }(\mathrm{Hz})\end{array}$ & $\begin{array}{c}\text { Equivalent viscous } \\
\text { damping }(\mathrm{N} \cdot \mathrm{s} / \mathrm{m})\end{array}$ & $\begin{array}{c}\text { Equivalent stiffness } \\
(\mathrm{N} / \mathrm{s})\end{array}$ \\
\hline 5.6 & 2.37847 & 2.527 & 1525 & 265 & 1062646 \\
16 & 0.86828 & 2.445 & 1715 & 659 & 2815978 \\
80 & 0.17324 & 2.377 & 2435 & 2369 & 13719903 \\
160 & 0.08665 & 2.366 & 2795 & 4129 & 27308918 \\
320 & 0.04329 & 2.360 & 3010 & 7670 & 54505874 \\
800 & 0.01733 & 2.357 & 3120 & 18506 & 136020960 \\
\hline
\end{tabular}

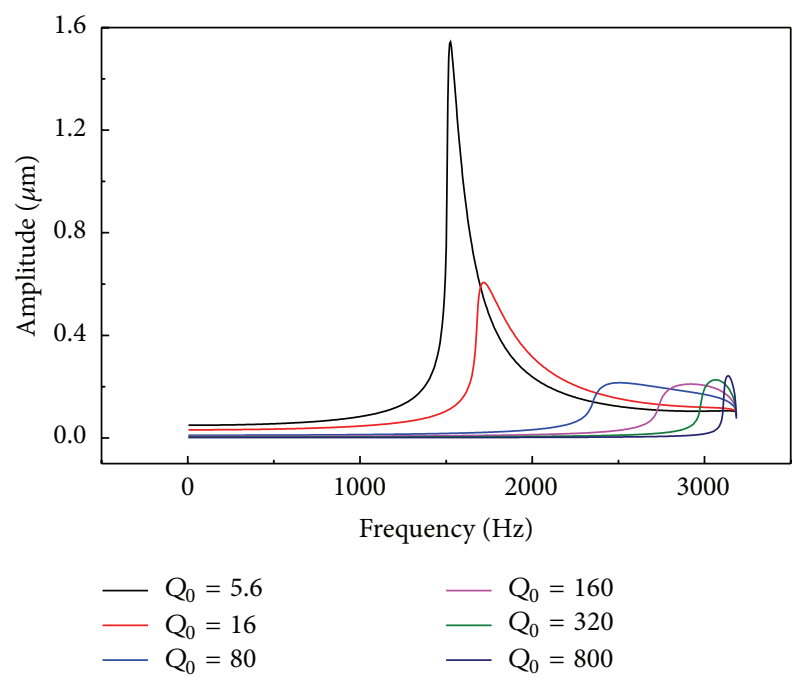

FIGURE 7: Vibration response of dry friction blade $m_{1}$ with shroud structure.

correlated with the stiffness. As a result, the resonance amplitude of $m_{1}$ decreases and the resonant frequency increases.

The resonance amplitude of $m_{2}$ decreases first and then increases along with the increase of positive pressure. Moreover, the changing trend of the resonance amplitude in Figure 6 is consistent with the conclusion as above. While the positive pressure $Q_{0}$ equals 320, the resonance amplitude reaches the minimum. This indicates that there exists optimal positive pressure which makes blade resonance response reach minimum for a blade with lacing structure. As the same as mass $m_{1}$, the resonance frequency of $m_{2}$ increases with the increase of positive pressure.

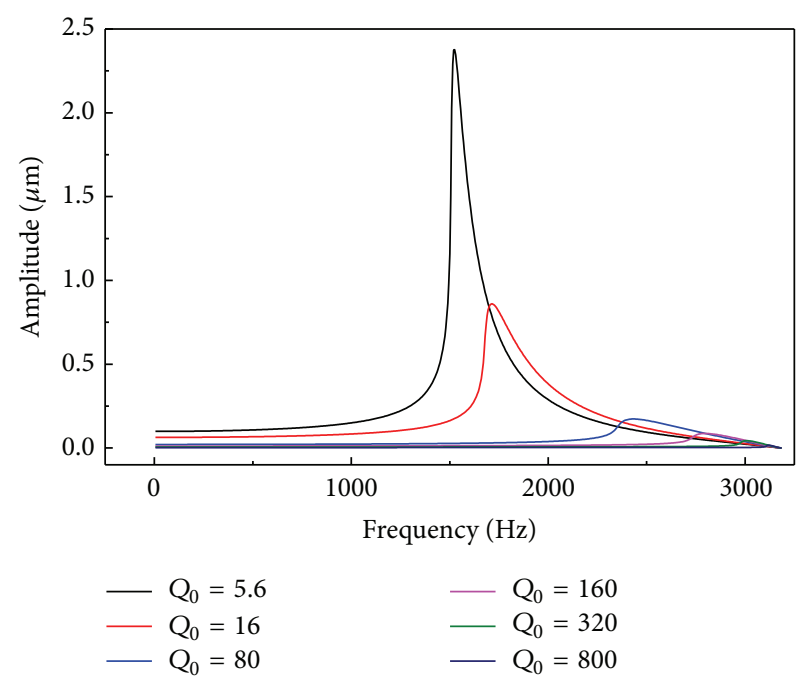

FIGURE 8: Vibration response of dry friction blade $m_{2}$ with shroud structure.

Comparing the amplitudes of the mass $m_{1}$ and $m_{2}$, the amplitude of $m_{2}$ is found greater than that of $m_{1}$ in calculation of all frequencies. In this case, the vibration inhibition effect towards $m_{1}$ that damping structure brought is much greater than the inhibition effect towards $m_{2}$.

The damper is placed on the mass $m_{2}$ to simulate shroud structure. Figures 7 and 8 present the amplitude-frequency response curves in the frequency range of $0 \sim 3200 \mathrm{~Hz}$ of $m_{1}$ and $m_{2}$ with shroud structure. Table 2 shows the resonance parameters of the damper under various positive pressures.

As shown in Figures 7 and 8, when the damper is placed on the mass $m_{2}$, the resonance amplitude of $m_{1}$ 
decreases first and then increases along with the increase of positive pressure. While the positive pressure $Q_{0}$ equals 160 , the resonance amplitude reaches the minimum. And the resonance frequency of $m_{1}$ increases gradually with the increase of positive pressure. On the other hand, the resonance amplitude of $m_{2}$ decreases with the increase of positive pressure. The resonance frequency of $m_{2}$ increases along with the increase of positive pressure.

Comparing the amplitude of $m_{1}$ and $m_{2}$, it is found that, when the positive pressure is smaller than or equal to 80 , the amplitude of $m_{2}$ is greater than the amplitude of $m_{1}$ and the difference between the two amplitudes is smaller than the difference obtained when the damper is placed on $m_{1}$. When the positive pressure is greater than 80 , the amplitude of $m_{1}$ is greater than that of $m_{2}$ while the frequency is near the resonance frequency. However, the amplitude of $m_{1}$ remains smaller than that of $m_{2}$ when the frequency is beyond this range. In this case, the vibration inhibition effect towards $m_{2}$ that damping structure brought is much greater than the inhibition effect towards $m_{1}$. When the positive pressure is relatively small, the inhibition effect could be small accordingly. But if the positive pressure is relatively large, particularly under the resonance state, the equivalent stiffness of the damper is very large and the inhibition effect towards $m_{2}$ would be great as well. This results in the fact that the amplitude of $m_{2}$ stays smaller than that of $m_{1}$ within certain range around the resonance frequency.

Comparing the results obtained when the damper is placed separately on $m_{1}$ and $m_{2}$, it is found that the resonance amplitude changing trend of $m_{1}$ in the first condition is the same as the changing trend of $m_{2}$ in the second condition. And the resonance amplitude changing trend of $m_{2}$ is the same as the changing trend of $m_{1}$ in the second condition. But, under the same positive pressure, the resonance frequency of $m_{2}$ with the damper is higher than that of $m_{1}$ with the damper. By the contrast of Tables 1 and 2, it is obvious that the equivalent stiffness of $m_{2}$ with the damper is much greater than that of $m_{1}$ with the damper. So, in the same situation, the resonance frequency of $m_{2}$ increases drastically.

The area surrounded by a hysteresis loop represents the energy dissipation amount in a vibration cycle of the damper. From Figures 9 and 10 and Table 3, it could be seen that, whether the damper is placed on $m_{1}$ or $m_{2}$, the energy dissipation amount of the damper decreases overall along with the increase of positive pressure. Based on formula (8), the energy dissipation amount in a vibration cycle of the damper is relevant to the positive pressure and the sliding part length $\delta_{a}$. The energy dissipation amount is positively correlated with the positive pressure and negatively correlated with the sliding part length.

Under the same condition, the consumed energy of vibration when the damper is placed on lacing is greater than that when the damper is placed on shroud structure. Comparing Tables 1 and 2 and Figures 9 and 10, it is found that, when the damper is placed on lacing structure, the maximum excitation force on the damper presents linear variation with the increase of pressure. And the excitation force on the damper increases as the positive pressure increases. When damper is placed on shroud structure, the maximum

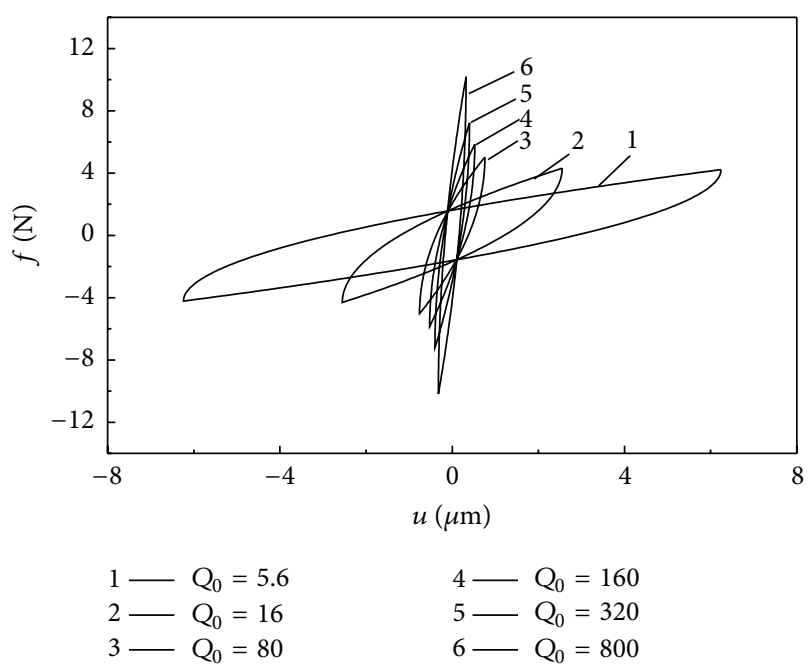

FIGURE 9: Resonance hysteresis loops of dry friction blade with lacing wire.

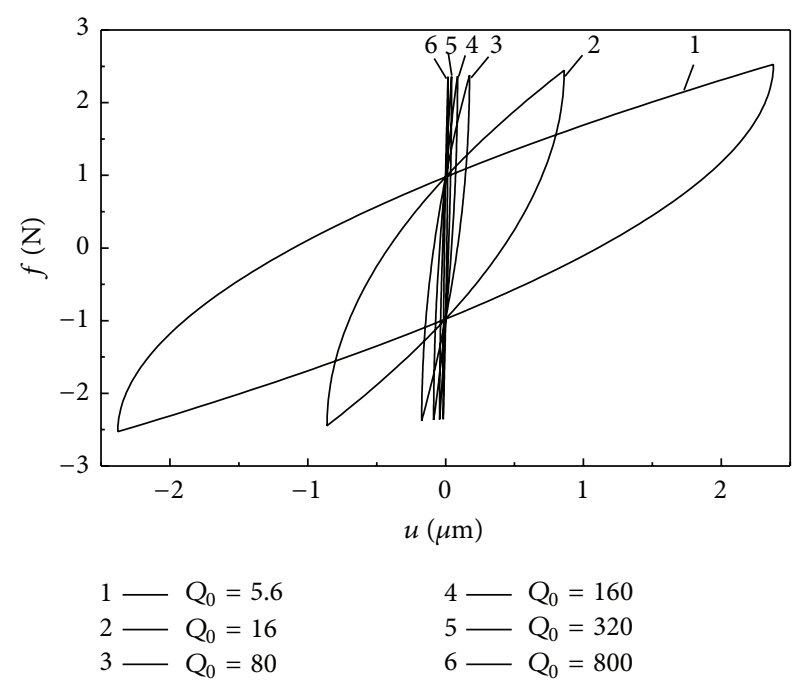

FIGURE 10: Resonance hysteresis loops of dry friction blade with shroud structure.

excitation force on the damper presents linear variation with the increase of pressure. The variation amplitude is pretty small and decreases as the positive pressure increases.

Assuming the placing of the damper on the blade root, as the vibration displacement amplitude at the root is close to zero, the consumed energy in a vibration cycle is less than the consumed energy at the lacing structure. Meanwhile, from Figures 9 and 10 and Table 3, it could be seen that, under the same positive pressure, the consumed energy when the damper is placed on lacing wire is greater than that while the damper is placed on shroud structure. Combining mathematical knowledge, it could be obtained that there existed one position so that, when the damper is placed on this position, the consumed energy in a vibration cycle reaches maximum. 
TABLE 3: Energy dissipation of the damper at resonance state.

\begin{tabular}{|c|c|c|c|c|c|c|}
\hline \multirow{2}{*}{$\begin{array}{l}\text { Pressure } Q_{0} \\
(\mathrm{~N})\end{array}$} & \multicolumn{3}{|c|}{ Damper located on $m_{1}$} & \multicolumn{3}{|c|}{ Damper located on $m_{2}$} \\
\hline & $\begin{array}{c}\text { Resonance } \\
\text { frequency }(\mathrm{Hz})\end{array}$ & $\begin{array}{c}\text { Sliding part length } \\
\delta_{a}(\mathrm{~m}) \\
\end{array}$ & $\begin{array}{l}\text { The dissipation of } \\
\text { energy } \times 10^{-6}(\mathrm{w})\end{array}$ & $\begin{array}{c}\text { Resonance } \\
\text { frequency }(\mathrm{Hz})\end{array}$ & $\begin{array}{c}\text { Sliding part length } \\
\delta_{a}(\mathrm{~m}) \\
\end{array}$ & $\begin{array}{l}\text { The dissipation of } \\
\text { energy } \times 10^{-6}(\mathrm{w})\end{array}$ \\
\hline 5.6 & 1420 & 0.1115447 & 30.4667 & 1525 & 0.0710186 & 7.1782 \\
\hline 16 & 1465 & 0.0451878 & 13.5863 & 1715 & 0.0271995 & 2.6746 \\
\hline 80 & 1630 & 0.0118769 & 4.9762 & 2435 & 0.0057784 & 0.5436 \\
\hline 160 & 1740 & 0.0070775 & 4.0425 & 2795 & 0.0029157 & 0.2720 \\
\hline 320 & 1855 & 0.0044180 & 3.8393 & 3010 & 0.0014642 & 0.1359 \\
\hline 800 & 1985 & 0.0025145 & 4.3451 & 3120 & 0.0005877 & 0.0544 \\
\hline
\end{tabular}

\section{Conclusions}

(1) Because of the complexity and self-similarity of contact surface geometry, contact surface can be described accurately using fractal dimension and fractal length.

(2) Whether shroud or lacing structure, it not only has the effect of dissipating energy but also changes blade stiffness. Meanwhile, in the same condition of pressure and contact area, the damping effect of lacing structure is stronger than that of shroud structure, but the stiffness effect is opposite.

(3) When damper is located on mass $m_{1}$, as pressure increases, the resonance frequencies of masses $m_{1}$ and $m_{2}$ increase. The resonance amplitude of mass $m_{1}$ decreases and the resonance amplitude of mass $m_{2}$ decreases first and then increases. It is obvious that there is an optimal pressure, making resonance amplitude minimum. Moreover, the resonance amplitude of $m_{1}$ is less than that of $m_{2}$.

(4) When damper is located on mass $m_{2}$, as pressure increases, the resonance frequencies of masses $m_{1}$ and $m_{2}$ increase. The resonance amplitude of mass $m_{1}$ decreases first and then increases while the resonance amplitude of mass $m_{2}$ decreases. There is an optimal pressure that makes resonance amplitude of mass $m_{1}$ minimum. When pressure is relatively small, the resonance amplitude of $m_{1}$ is smaller than that of $m_{2}$. When pressure is relatively large, near the resonance frequency, there is a frequency range that makes resonance amplitude of $m_{1}$ bigger than that of $m_{2}$.

(5) Under the same circumstance, resonance frequency is lower when damper is located on $m_{1}$ than when it is located on $m_{2}$, but the vibration amplitude is opposite.

(6) The damper consumes more energy when it is located on mass $m_{1}$ than the situation when it is located on $m_{2}$. No matter which mass damper is located, the energy consumed in a vibration cycle declined as the pressure increases.

(7) Under the same circumstance, there is at least one position on the blade, making damper energy consumption maximum in one vibration cycle.

\section{Conflict of Interests}

The authors declare that there is no conflict of interests regarding the publication of this paper.

\section{References}

[1] M. E. Ritchie and H. Olff, "Spatial scaling laws yield a synthetic theory of biodiversity," Nature, vol. 400, no. 6744, pp. 557-560, 1999.

[2] R. P. Taylor, A. P. Micolich, and D. Jonas, "Fractal analysis of Pollock's drip paintings," Nature, vol. 399, no. 6735, p. 422, 1999.

[3] J. P. Den Hartog, "Forced vibrations with combined Coulomb and viscous friction," Transactions of the American Society of Mechanical Engineers, vol. 53, no. 9, pp. 107-115, 1931.

[4] C.-H. Meng, J. Bielak, and J. H. Griffin, "The influence of microslip on vibratory response, part I: a new microslip model," Journal of Sound and Vibration, vol. 107, no. 2, pp. 279-293, 1986.

[5] C.-H. Meng and P. Chidamparam, "Friction damping of twodimensional motion and its application in vibration control," Journal of Sound and Vibration, vol. 144, no. 3, pp. 427-447, 1991.

[6] A. Muszyńska and D. I. G. Jones, "On tuned bladed disk dynamics: some aspects of friction related mistuning," Journal of Sound and Vibration, vol. 86, no. 1, pp. 107-128, 1983.

[7] A. Muszynska, D. I. G. Jones, T. Lagnese, and L. Whitford, "On nonlinear response of multiple blade systems," Shock and Vibration Bulletin, vol. 51, no. 3, pp. 89-110, 1981.

[8] D. I. G. Jones and A. Muszynska, "Design of turbine blades for effective slip damping at high rotational speeds," Shock and Vibration Bulletin, vol. 49, pp. 87-96, 1979.

[9] G. Csaba, Modelling Microslip Friction Damping and Its Influence on Turbine Blade Vibrations, Division of Machine Design Department of Mechanical Engineering, Linköping University, 1998.

[10] Y. Liu, B. Shangguan, and Z. Xu, "A friction contact stiffness model of fractal geometry in forced response analysis of a shrouded blade," Nonlinear Dynamics, vol. 70, no. 3, pp. 22472257, 2012. 


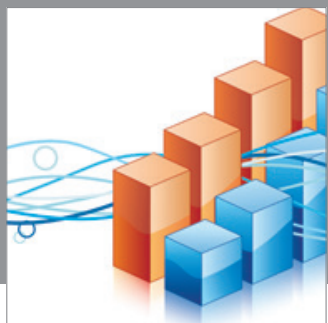

Advances in

Operations Research

mansans

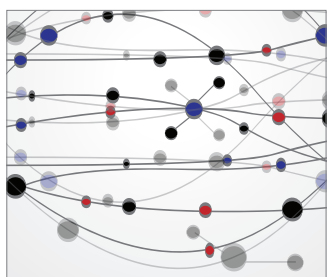

The Scientific World Journal
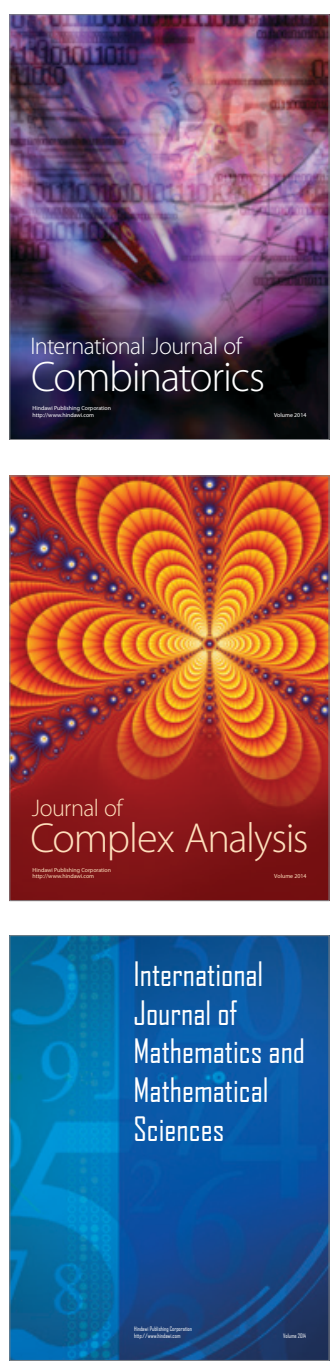
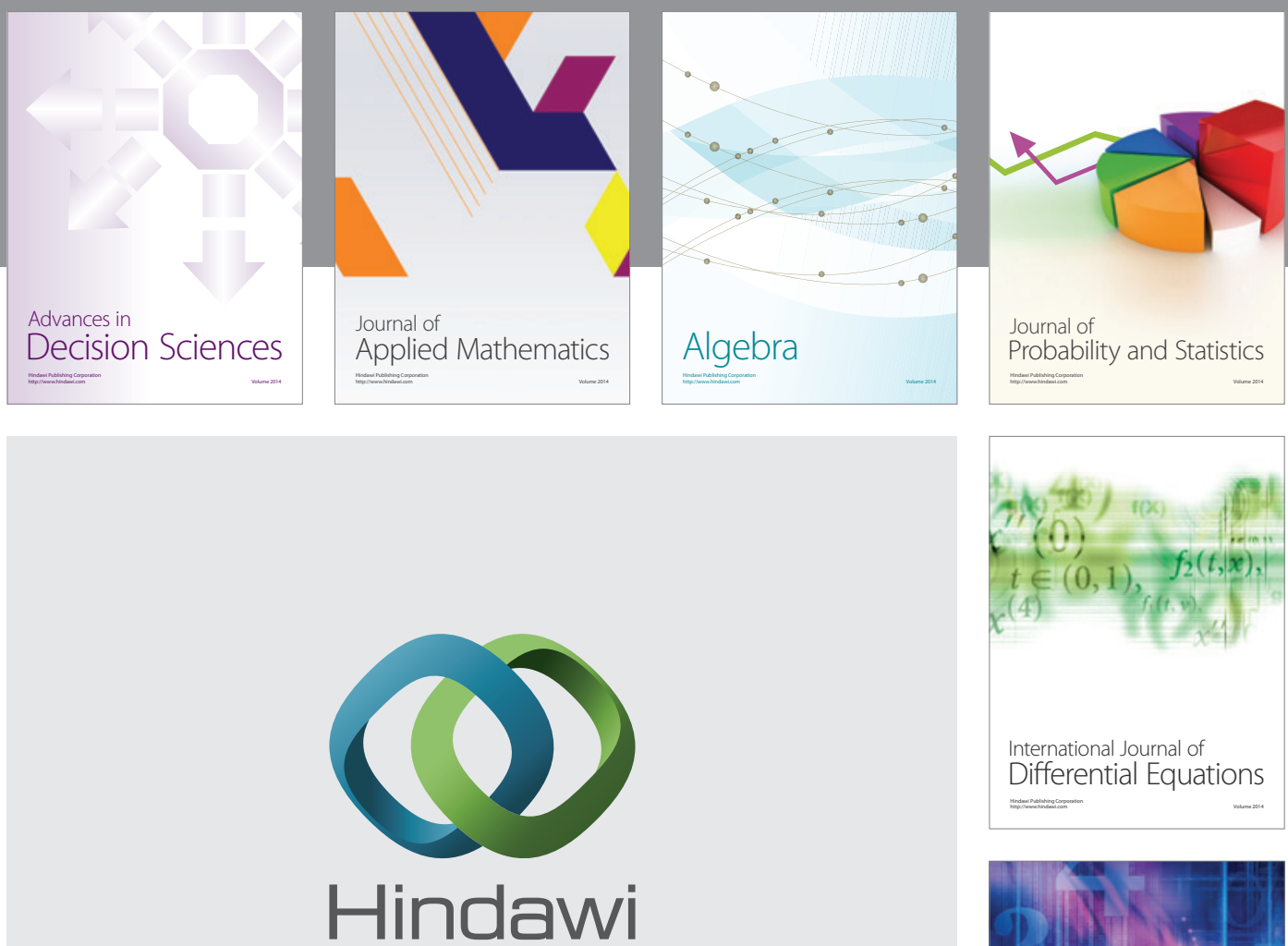

Submit your manuscripts at http://www.hindawi.com
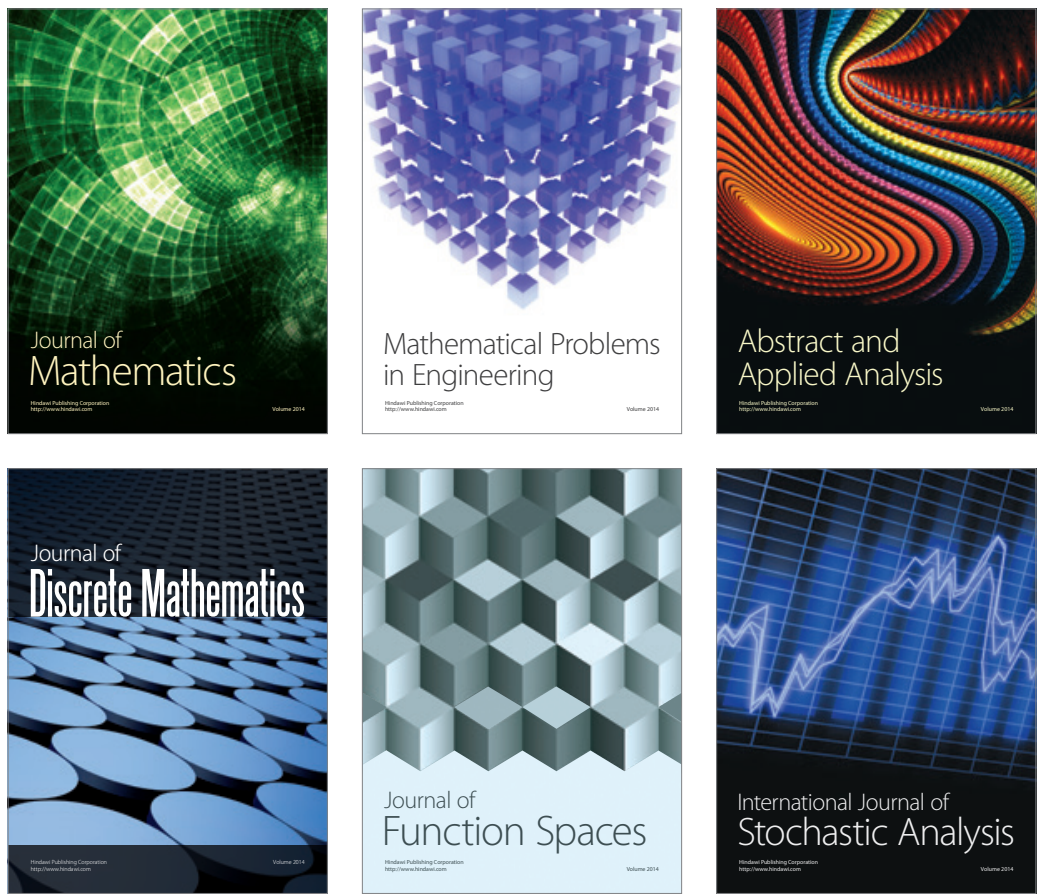

Journal of

Function Spaces

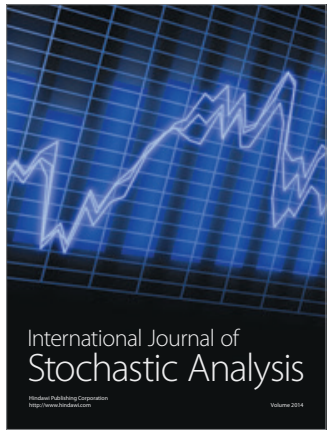

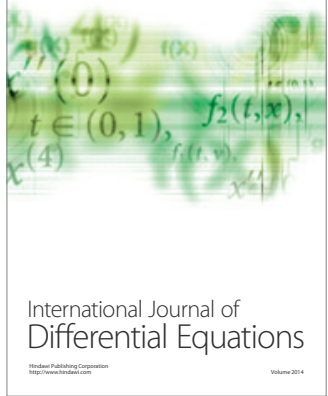
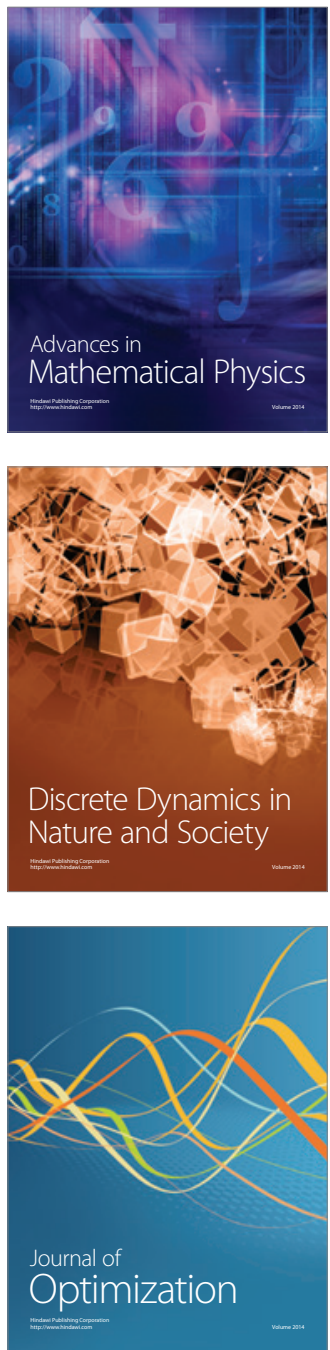